\title{
Multi-agent system for dynamic scheduling
}

\author{
$1^{\text {st }}$ Bernardo Firme \\ IDMEC, Instituto Superior Técnico \\ Universidade de Lisboa \\ Lisbon, Portugal \\ bernardo.firme@tecnico.ulisboa.pt \\ $4^{\text {th }}$ Tiago Coito \\ IDMEC, Instituto Superior Técnico \\ Universidade de Lisboa \\ Lisbon, Portugal \\ tiagoascoito@tecnico.ulisboa.pt \\ $7^{\text {th }}$ João C. P. Reis \\ IDMEC, Instituto Superior Técnico \\ Universidade de Lisboa \\ Lisboa, Portugal \\ joao.c.p.reis@tecnico.ulisboa.pt
}

\author{
$2^{\text {nd }}$ Guilherme Lopes \\ Instituto Superior Técnico \\ Universidade de Lisboa \\ Lisbon, Portugal
}

guilherme.cordeiro.lopes@tecnico.ulisboa.pt

\author{
$5^{\text {th }}$ Joaquim Viegas \\ IDMEC, Instituto Superior Técnico \\ Universidade de Lisboa \\ Lisbon, Portugal \\ joaquim.viegas@tecnico.ulisboa.pt
}

$8^{\text {th }}$ João Figueiredo

Department of Physics

Universidade de Évora

Évora, Portugal

jfig@uevora.pt

\author{
$3^{\text {rd }}$ Miguel S. E. Martins \\ IDMEC, Instituto Superior Técnico \\ Universidade de Lisboa \\ Lisbon, Portugal \\ miguelsemartins@tecnico.ulisboa.pt
}

\author{
$6^{\text {th }}$ João M. C. Sousa \\ IDMEC, Instituto Superior Técnico \\ Universidade de Lisboa \\ Lisbon, Portugal \\ jmsousa@tecnico.ulisboa.pt \\ $9^{\text {th }}$ Susana Vieira \\ IDMEC, Instituto Superior Técnico \\ Universidade de Lisboa \\ Lisbon, Portugal \\ susana.vieira@tecnico.ulisboa.pt
}

\begin{abstract}
This paper proposes a flexible manufacturing system based on intelligent computational agents. A Multi-Agent System composed of 4 types of reactive agents was designed to control the operation of a real implementation in the Intelligent Automation Lab at Instituto Superior Técnico. This implementation was based and constructed analogously to a known benchmark, AIPPRIMECA. The agents were modelled using Petri nets and agent communications were defined through the combination of FIPA Interaction Protocols. The system was tested under the conditions of static and dynamic scenarios, having its performance validated whenever possible by comparison with results from a Potential Fields Approach in the same benchmark. Overall, the performance exhibited by the proposed MAS was slightly better and it is worth highlighting the simple behaviour of each agent and ability to respond in real-time to all the dynamic scenarios tested.

Index Terms-Multi-Agent Systems, Agent Communication,
\end{abstract} Manufacturing Control, Flexible Manufacturing System

\section{INTRODUCTION}

The industry has been evolving to provide products with increasingly high-quality and customization, having already faced three revolutionary stages known as industrial revolutions. With the introduction into the manufacturing world of emerging technologies like Internet of Things, wireless sensor networks, Big Data, cloud computing and Artificial Intelligence, a new paradigm arrived to the industry world: the fourth industrial revolution [1].

Aiming the achievement of a smart, flexible and reconfigurable factory, capable of producing customized and smalllot products efficiently and profitably, the use of traditional manufacturing control systems is not enough. These control

This work was supported by FCT, through IDMEC, under LAETA, project UIDB/50022/2020 systems do not exhibit sufficient capabilities of responsiveness, flexibility and reconfigurability, since they are designed based on centralized and hierarchical control structures that, despite presenting good production optimization, present insufficient response to change due to rigidity and centralization. To respond to this handicaps and taking advantage of the emergent technologies, some advanced manufacturing control systems have been proposed to achieve the smart factory of the future. [2]

A representative case of the advanced manufacturing control systems is the agent-based manufacturing control based on Multi-Agent Systems (MAS) technology. MAS consist in an ecosystem of manufacturing resources defined as intelligent, autonomous and cooperative computational entities, known as agents, that can negotiate with each other to implement decision making and dynamical reconfiguration, in order to achieve their individual goals which, in aggregate, accomplish an overall objective. In an agent-based manufacturing control system, all the agents are in the same hierarchy level, being organized in a autonomous, distributed and decentralized architecture [2].

Throughout the last decades, several different approaches, architectures and platforms regarding MAS have been introduced and a considerable amount of industrial applications were already implemented. The main fields of application have been smart production, smart electric grids, smart logistics and smart healthcare [3], although some authors also have a prospect of other fields that might benefit from the application of agent technologies, namely traffic control, buildings and home automation, military and network security [4]. Some recent application of MAS can be found in [16] for optimal energy management and in [17] for management of traffic 